\title{
The evolutionary status of the bright high-latitude supergiant HD 190390^
}

\author{
M. Reyniers ${ }^{1, \star \star}$ and J. Cuypers ${ }^{2}$ \\ 1 Instituut voor Sterrenkunde, Departement Natuurkunde en Sterrenkunde, KU Leuven, Celestijnenlaan 200B, \\ 3001 Leuven, Belgium \\ e-mail: Maarten.Reyniers@ster.kuleuven.ac.be \\ 2 Koninklijke Sterrenwacht van België, Ringlaan 3, 1180 Brussel, Belgium
}

Received 30 September 2004 / Accepted 31 October 2004

\begin{abstract}
Despite its mean apparent magnitude of $m_{\mathrm{V}}=6.39$, the evolutionary status of HD 190390 (HR 7671), a luminous F-type supergiant at high galactic latitude, is still not very clear, but in most papers a post-AGB classification is assumed. New observational material has been obtained with four different instruments and is presented here. An extensive abundance analysis based on high resolution, high signal-to-noise NTT+EMMI spectra confirms the metal deficiency of this object $([\mathrm{Fe} / \mathrm{H}]=-1.6)$, together with a high lithium content $(\log \epsilon(\mathrm{Li})=1.9)$. A variability analysis based on Geneva photometry over seven years reveals beating with a period of $\sim 3000$ days. It is, however, not clear whether this beating is caused by a stable triplet, or it is the consequence of small changes in the main frequency. More recent data obtained with the HIPPARCOS satellite and the Mercator telescope not only confirm the main period, but also support the presence of a second periodicity of 11 days, which was also found in the Geneva photometry. A conclusive evolutionary status of this object is not given, but alternative to the UU Her (i.e. post-AGB) status, a W Vir classification is discussed.
\end{abstract}

Key words. stars: abundances - stars: AGB and post-AGB - stars: evolution - stars: individual: HD 190390 - stars: oscillations stars: Population II

\section{Introduction}

Since their discovery by William Bidelman (1951), the evolutionary stage of HR $6144\left(b=+25^{\circ}\right), 89 \operatorname{Her}\left(+22^{\circ}\right)$ and HD $161796\left(+30^{\circ}\right)$, three high-luminosity stars at moderately high galactic latitude, has been a matter of debate. At least three different possibilities were suggested: (1) young, massive objects that recently escaped from a star forming region; (2) low-mass, evolved objects; or (3) a rare product in a binary star scenario. After the high resolution, high signal-tonoise spectroscopic study of Luck et al. (1990), there was general consensus that these objects are low-mass objects in an evolved, post-AGB evolutionary stage. Although no s-process enhancement was observed in these stars, there were enough indications for a classification as a post-AGB star (slightly metal-poor, overabundant carbon and oxygen and a strong infrared dust excess indicating high mass loss in a previous evolutionary (AGB) stage).

There was also a fourth star in the Luck et al. sample, HD 190390 (HR 7671, Table 1). The inclusion of this object in

^ Based on observations collected at the European Southern Observatory, La Silla, Chile (programme 61.E-0426), and at the Observatorio del Roque de los Muchachos, La Palma, Spain.

$\star \star$ Postdoctoral fellow of the Fund for Scientific Research, Flanders.
Table 1. Basic parameters of HD 190390 (SIMBAD database).

\begin{tabular}{|c|c|c|}
\hline \multicolumn{3}{|c|}{ HD 190390 (HR 7671) } \\
\hline \multirow[t]{2}{*}{ Coordinates } & $\alpha_{2000}$ & 200505.41 \\
\hline & $\delta_{2000}$ & -113557.9 \\
\hline Galactic & $l$ & 30.60 \\
\hline coordinates & $b$ & -21.53 \\
\hline \multirow[t]{2}{*}{ Mean magnitude } & $B$ & 6.88 \\
\hline & $V$ & 6.39 \\
\hline Spectral type* & & F1III \\
\hline Parallax & $\pi$ (mas) & $2.56 \pm 0.97$ \\
\hline IRAS fluxes & $f_{12}$ & 0.65 \\
\hline \multirow[t]{3}{*}{$(\mathrm{Jy})$} & $f_{25}$ & $<0.32$ \\
\hline & $f_{60}$ & $<0.40$ \\
\hline & $f_{100}$ & $<1.08$ \\
\hline
\end{tabular}

*Very differing spectral types have been reported in the literature: $\mathrm{gF} 4$, F1III, F6II, F6Ib, F3Ib, F2p (shell) (cit. Fernie 1986).

their sample was motivated not only by its similar galactic latitude and spectral type, but also by the variability pointed out by 
Waelkens \& Burnet (1985) and Fernie (1986). This variablity made HD 190390 a candidate member of the UU Her type stars, a heterogeneous class of luminous variables at high galactic latitudes defined by Sasselov (1984), which also includes $89 \mathrm{Her}$ and HD 161796. For HD 190390, however, Luck et al. (1990) found a clearly different chemical signature than for the other three stars, with a $\mathrm{C}$ and $\mathrm{O}$ deficiency and a high $\mathrm{Li}$ abundance. The authors proposed the object to be a descendant of a Li-rich S-type star, with the Li attributed to a "hot bottom burning" event.

In this paper, new observational material is presented to clarify the status of this intriguing object. Both the chemical composition and the variability are reanalysed, based on high quality data coming from different instruments and telescopes. In Sect. 2 we report on the reduction and analysis of our high resolution, high signal-to-noise NTT+EMMI spectra and discuss the chemical photospheric composition of HD 190390. In Sect. 3, a detailed variability analysis is presented, based on both photometric data and radial velocities. Some results are presented originating from the new Flemish Mercator telescope located at La Palma, Spain. Our results are discussed in Sect. 4. We give our conclusions in Sect. 5.

\section{Chemical composition}

\subsection{Previous studies}

Luck et al. (1990) were the first to carry out a detailed abundance analysis of HD 190390, based on spectra with medium to high resolution $(R=\lambda / \delta \lambda \simeq 15000-47000)$ and a signalto-noise ratio between 75 and 100. They confirmed the metal deficiency discovered by McDonald (1976) and quantified it: $[\mathrm{Fe} / \mathrm{H}]=-1.1$. Further, they found a slight $\mathrm{C}$ and $\mathrm{O}$ deficiency and also claimed a slight s-process enhancement by a factor of 4 above solar. Lithium was also detected with an abundance of $\log \epsilon(\mathrm{Li})=2.4$. The main shortcomings of this analysis were the quite low signal-to-noise ratio obtained, and the inhomogeneous collection of spectra with different resolution, leading to quite large line-to-line scatters of the reported abundances (up to $0.92 \mathrm{dex}$ ). A more recent abundance analysis was presented by Giridhar et al. (1997). This analysis was based on moderately high resolution spectra $(R \simeq 30000)$ with a rather narrow wavelength coverage (5260-5600 $\AA$ ), and it is therefore rather limited.

In the analysis we present here, we avoided the shortcomings of the previous studies: a large spectral domain covered with the same resolution and a high signal-to-noise was used. Such spectra were taken with ESO-NTT telescope and the EMMI spectrograph.

\subsection{Observations and reduction}

In the framework of our ongoing program to study the photospheric chemical composition of stars in their last stages of evolution (e.g. Van Winckel 2003; Reyniers et al. 2004), high resolution, high signal-to-noise spectra were taken with the EMMI Spectrograph mounted on the $3.58 \mathrm{~m}$ New Technology Telescope (NTT) located in La Silla, Chile. The observations
Table 2. Log of the high resolution, high signal-to-noise NTT+EMMI spectra of HD 190390. The resolving power $\lambda / \delta \lambda$ was $\sim 60000$ for both settings.

\begin{tabular}{ccrc}
\hline \hline Date and UT & $\begin{array}{c}\text { EMMI } \\
\text { setting }\end{array}$ & $\begin{array}{c}\text { Integration } \\
\text { time (s) }\end{array}$ & $\begin{array}{c}\text { Sp. range } \\
(\mathrm{nm})\end{array}$ \\
\hline 29/09/1998 02:55 & Ech\#14 Grsm\#5 & 900 & $398-662$ \\
30/09/1998 04:18 & Ech\#14 Grsm\#6 & 1200 & $597-832$ \\
\hline
\end{tabular}

of HD 190390 were made by Dr. H. Van Winckel during two nights in 1998 (see Table 2). The reduction of the data was performed in the specific echelle package ECHELLE of the MIDAS data analysis system. For a more detailed description of the different steps of the reduction procedure, see Reyniers (2002). A sample spectrum can be found in Fig. 1.

\subsection{Abundance analysis}

A detailed abundance analysis of HD 190390 was performed with an extended version of the line list described in Van Winckel \& Reyniers (2000).

\subsubsection{Atmospheric parameters and equivalent widths}

Since the atmospheric parameters are usually based on a fine analysis of the iron lines, the Van Winckel \& Reyniers list only contains the critical compilation of Lambert (Lambert et al. 1996), completed with values of Blackwell et al. (1980) for the singly ionised iron lines. Two different methods were used to measure the equivalent widths: a Gaussian approximation and direct integration. Due to a small asymmetry in the blue wing of the stronger lines, a Gaussian approximation leads to an underestimation of the real equivalent width. This is illustrated in the top panels of Fig. 2. On the other hand, a Gaussian approximation allows a better continuum placement (especially important for weaker lines) and also allows for blends in the wing of a line. We have chosen a strictly homogeneous way of measuring the equivalent widths of the iron lines: for lines with $W_{\lambda}<10.5 \mathrm{~m} \AA$, we applied a Gaussian approximation, for the other lines we used direct integration, except if the line was blended in the wings. $84 \mathrm{Fe}$ I lines and $24 \mathrm{Fe}$ II lines were measured in such way.

The effective temperature $T_{\text {eff }}$ of the Kurucz model atmosphere (Kurucz 1993) was then obtained by forcing the Fe abundance to be independent of the excitation potential of the lines, the gravity $\log g$ by forcing ionisation equilibrium, the microturbulent velocity $\xi_{\mathrm{t}}$ by forcing the $\mathrm{Fe}$ abundance to be independent of (reduced) equivalent widths. Abundance calculations were made with the LTE line analysis program MOOG (version April 2002). This iterative process finally yielded a model with $\left(T_{\text {eff }}, \log g, \xi_{\mathrm{t}},[\mathrm{M} / \mathrm{H}]\right)=(6250 \mathrm{~K}, 1.0(\mathrm{cgs})$, $\left.3.0 \mathrm{~km} \mathrm{~s}^{-1},-1.5\right)$. E.P. - abundance and $\log \left(W_{\lambda} / \lambda\right)$ - abundance diagrams for this model can be found in Fig. 2. The model parameters slightly differ from the model parameters found by Luck et al. (1990) and Giridhar et al. (1997). The former authors found $\left(6600 \mathrm{~K}, 1.5(\mathrm{cgs}), 2.3 \mathrm{~km} \mathrm{~s}^{-1}\right)$, the latter 


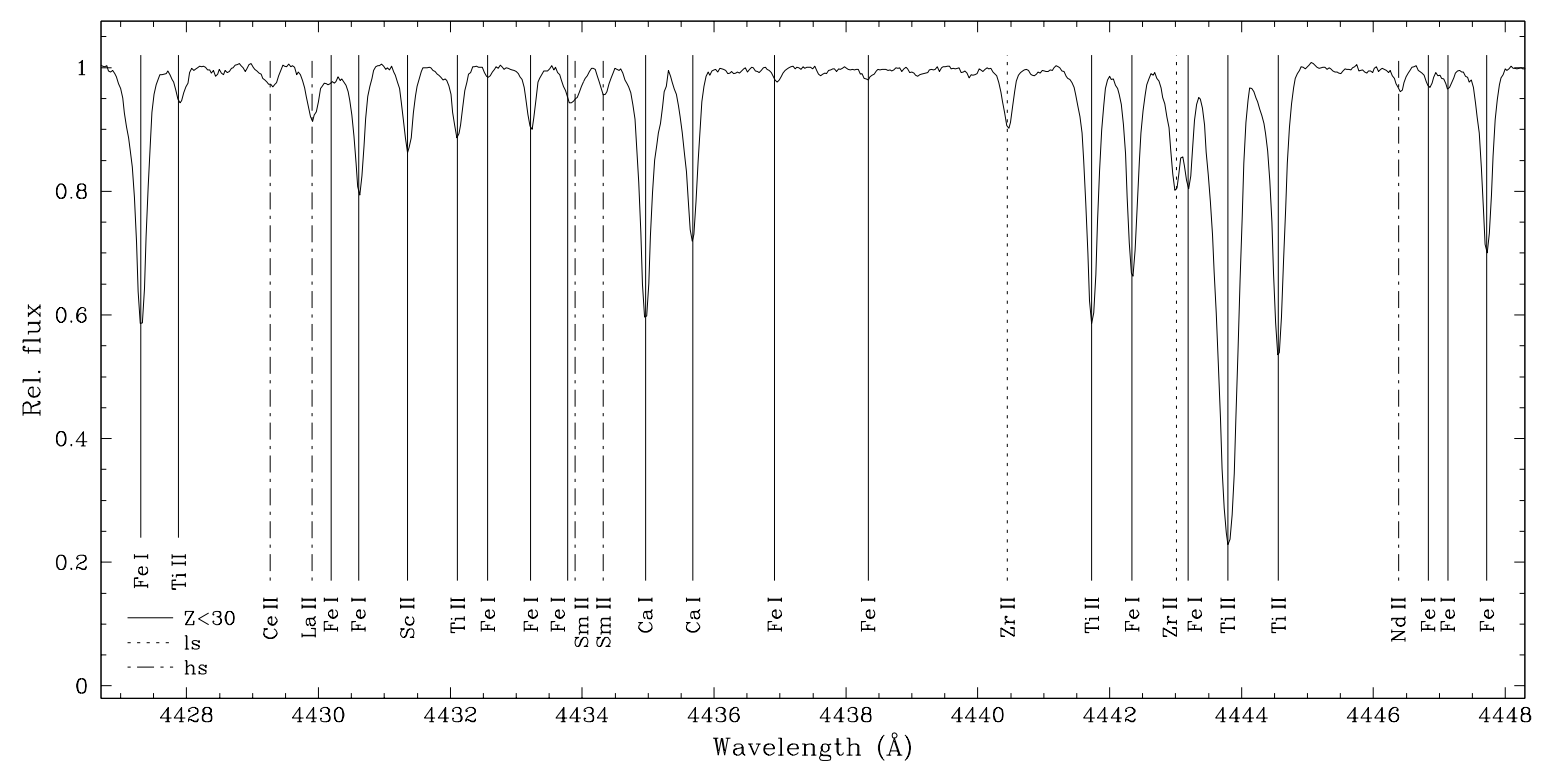

Fig. 1. The spectrum of HD 190390 between 4425 and $4450 \AA$ A. A complete line identification of this spectral interval has been attempted by the use of the VALD database (Kupka et al. 1999). Lines of light s-process elements (Sr peak) are shown with a dotted line; lines of heavy s-process elements (Ba peak) with a dash-dotted line; lines of other elements (which are mainly $\alpha$ and iron peak elements) with a full line. The signal-to-noise ratio of our spectra is around 500 .

$\left(6500 \mathrm{~K}, 1.25(\mathrm{cgs}), 3.2 \mathrm{~km} \mathrm{~s}^{-1}\right)$. However, due to the superior quality of our spectra and the severe selection of our Fe line list, we feel confident about our slightly differing parameters. Note that the difference in atmospheric parameters can also be a consequence of the variability of the object.

The method of the equivalent width measurement (either Gaussian approximation or direct integration) for the other lines was chosen for each line individually, without a strict criterion. The Van Winckel \& Reyniers list was extended for the s-process elements in the following way. We extracted all s-process elements from the VALD database (Kupka et al. 1999). These lists were then sorted based on the calculated equivalent widths of the lines using the model obtained above and an ad-hoc abundance value. Each line of these lists was then checked in this order on the spectrum of HD 190390, until the lines were too small to detect. 136 lines of s-process elements were used in the analysis. The oscillator strengths of La and Eu from VALD were replaced by the more recent values published by Lawler et al. (2001a,b) respectively. The total number of spectral lines used in this analysis is 462 . The line list can be obtained from the authors upon request.

\subsubsection{Abundance results}

The resulting abundances are given in Table 3. For each ion we list the number of lines $\mathrm{N}$, the mean equivalent width $\overline{W_{\lambda}}$, the absolute abundance $\log \epsilon$ (i.e. relative to $\mathrm{H}: \log \epsilon=\log X / \mathrm{H}+$ 12 ), the internal scatter $\sigma$, if more than one line is used, the solar abundances and the abundance ratio relative to iron [el/Fe]. The latter values are also graphically presented in the top panel of Fig. 3. For the solar iron abundance we used the meteoritic iron abundance of 7.51; the references for the solar CNO are C: Biémont et al. (1993), N: Hibbert et al. (1991) and O: Biémont et al. (1991); for Mg and Si the latest Holweger
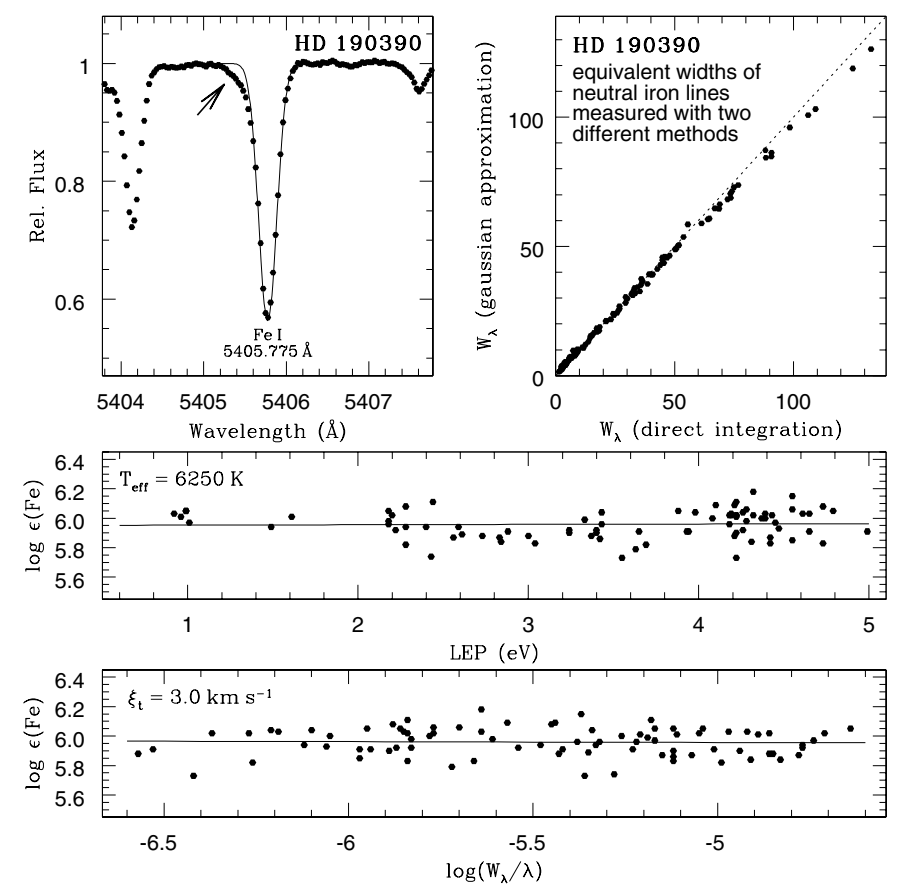

Fig. 2. Lines of neutral iron in the spectrum of HD 190390. The small asymmetry in the blue wings of the stronger lines makes a Gaussian approximation for these lines not acceptable. This is illustrated in the upper panels of the figure. Taking the Gaussian equivalent widths would lead to a wrong $\xi_{\text {t }}$. The two lower panels are E.P.-abundance and $\log \left(W_{\lambda} / \lambda\right)$-abundance diagrams for the final model of HD 190390.

values (Holweger 2001) were used; for $\mathrm{La}$ and $\mathrm{Eu}$ we took the recent values derived by Lawler et al. (2001a) and Lawler et al. (2001b) respectively; other solar abundances were taken from the review by Grevesse \& Sauval (1998). Despite the fact 
Table 3. Abundance results for HD 190390. The references for the solar abundances are given in the text (Sect. 2.3.2).

\begin{tabular}{|c|c|c|c|c|c|c|}
\hline & & & $\begin{array}{l}\text { HD } 190 \\
\text { eff }=625 \\
g=1.0 \\
\xi_{\mathrm{t}}=3.0\end{array}$ & $\begin{array}{l}90 \\
\mathrm{~K} \\
\mathrm{cgs}) \\
\mathrm{cm} \mathrm{s}^{-1}\end{array}$ & & \\
\hline Ion & $N$ & $\overline{W_{\lambda}}$ & $\log \epsilon$ & $\sigma$ & Sun & {$[\mathrm{el} / \mathrm{Fe}]$} \\
\hline $\mathrm{Li} \mathrm{I}$ & 1 & ss & 1.9 & 0.2 & & \\
\hline C I & 9 & 4 & 6.44 & 0.13 & 8.57 & -0.57 \\
\hline $\mathrm{NI}_{\mathrm{I}}$ & 3 & 5 & 6.94 & 0.16 & 7.99 & 0.51 \\
\hline O I & 3 & 5 & 7.76 & 0.05 & 8.86 & 0.46 \\
\hline $\mathrm{NaI}$ & 2 & 11 & 4.65 & 0.12 & 6.33 & -0.12 \\
\hline Mg I & 3 & 91 & 6.47 & 0.09 & 7.54 & 0.49 \\
\hline Mg II & 1 & 8 & 6.46 & & 7.54 & 0.48 \\
\hline Si I & 17 & 11 & 6.50 & 0.12 & 7.54 & 0.52 \\
\hline Si II & 5 & 46 & 6.47 & 0.17 & 7.54 & 0.49 \\
\hline S I & 5 & 9 & 6.06 & 0.06 & 7.33 & 0.29 \\
\hline $\mathrm{Ca} \mathrm{I}$ & 23 & 36 & 5.10 & 0.13 & 6.36 & 0.30 \\
\hline Sc II & 13 & 54 & 1.72 & 0.12 & 3.17 & 0.11 \\
\hline Ti I & 2 & 27 & 3.80 & 0.01 & 5.02 & 0.34 \\
\hline Ti II & 42 & 76 & 3.82 & 0.10 & 5.02 & 0.36 \\
\hline V II & 1 & 96 & 2.66 & & 4.00 & 0.22 \\
\hline $\mathrm{Cr} \mathrm{I}$ & 16 & 18 & 4.07 & 0.13 & 5.67 & -0.04 \\
\hline Cr II & 23 & 28 & 4.01 & 0.11 & 5.67 & -0.10 \\
\hline Mn I & 9 & 9 & 3.52 & 0.10 & 5.39 & -0.31 \\
\hline $\mathrm{Fe} \mathrm{I}$ & 84 & 30 & 5.96 & 0.10 & 7.51 & 0.01 \\
\hline Fe II & 24 & 41 & 5.95 & 0.12 & 7.51 & 0.00 \\
\hline Ni I & 35 & 10 & 4.78 & 0.10 & 6.25 & 0.09 \\
\hline Ni II & 2 & 7 & 4.79 & 0.01 & 6.25 & 0.10 \\
\hline $\mathrm{Cu} \mathrm{I}$ & 1 & 3 & 2.56 & & 4.21 & -0.09 \\
\hline $\mathrm{Zn} \mathrm{I}$ & 3 & 19 & 3.25 & 0.03 & 4.60 & 0.21 \\
\hline Y II & 22 & 34 & 0.80 & 0.13 & 2.24 & 0.12 \\
\hline $\mathrm{Zr}$ II & 31 & 35 & 1.48 & 0.15 & 2.60 & 0.44 \\
\hline Ba II & 3 & 30 & 0.63 & 0.11 & 2.13 & 0.06 \\
\hline La II & 16 & 19 & -0.21 & 0.09 & 1.13 & 0.22 \\
\hline Ce II & 32 & 11 & 0.15 & 0.22 & 1.58 & 0.13 \\
\hline Nd II & 14 & 10 & 0.07 & 0.16 & 1.50 & 0.13 \\
\hline Sm II & 15 & 6 & -0.41 & 0.13 & 1.01 & 0.14 \\
\hline Eu II & 2 & ss & -0.71 & 0.22 & 0.52 & 0.33 \\
\hline Hf II & 1 & 4 & -0.43 & & 0.88 & 0.25 \\
\hline \multicolumn{7}{|c|}{$[\mathrm{Fe} / \mathrm{H}]=-1.56$} \\
\hline \multicolumn{7}{|c|}{$\mathrm{C} / \mathrm{O}=0.05$} \\
\hline \multicolumn{7}{|c|}{$[\alpha / \mathrm{Fe}]=+0.4(\alpha: \mathrm{Mg}, \mathrm{Si}, \mathrm{S}, \mathrm{Ca}, \mathrm{Ti})$} \\
\hline
\end{tabular}

that there are more recent values for some of the solar abundances (especially for the solar CNO), we take these references to ensure as much as possible that the $g f$ values that we have
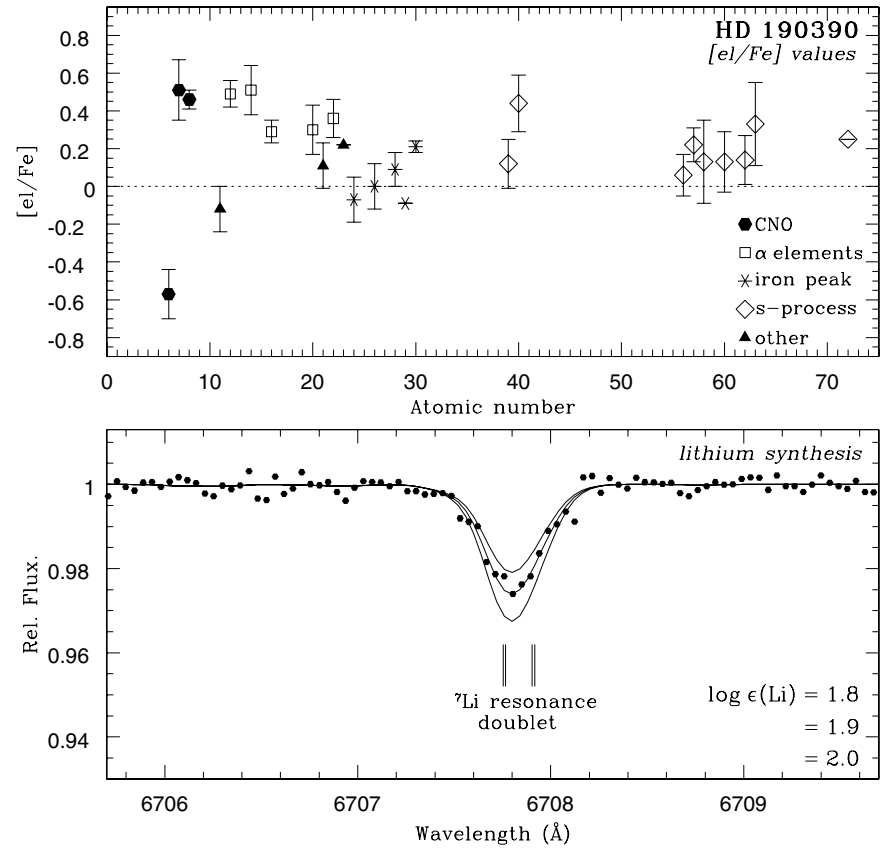

Fig. 3. $[\mathrm{el} / \mathrm{Fe}]$ values (upper panel) and lithium doublet synthesis (lower panel) for HD 190390. The error on the $[\mathrm{el} / \mathrm{Fe}]$ values is the line-to-line scatter found in Table 3.

used in the present paper are consistent with the adopted solar abundances. Note the excellent agreement in abundance for elements with two ionisation stages, $\mathrm{Mg}, \mathrm{Si}, \mathrm{Ti}, \mathrm{Cr}$ and $\mathrm{Ni}$, indicating a good model parameter choice. Note also the small line-to-line scatter which rarely exceeds $0.15 \mathrm{dex}$, including the scatter for the s-process ions.

\subsubsection{Lithium synthesis}

The lithium line in HD 190390 with equivalent width $W_{\lambda}=$ $9.5 \mathrm{~m} \AA$ was first reported by Luck et al. (1990). To account for the doublet structure of the line, spectrum synthesis is necessary in deriving the Li abundance. The program MOOG was also used for this purpose. The line list is identical to the line list that was used in Reyniers et al. (2002), but due to the combined effect of the quite high temperature and the metal deficiency of HD 190390, no other lines are detectable in the 6707 Å region.

For a spectral synthesis, a broadening factor is needed, in addition to the model atmosphere parameters. This broadening factor is a combined effect of the instrumental, rotational and macroturbulent broadening. An instrumental broadening of $5 \mathrm{~km} \mathrm{~s}^{-1}$ was adopted (the spectral resolution of the EMMI spectrograph). The rotational broadening is thought not to be very high due to the supergiant character of HD 190390. The macroturbulent broadening $\xi_{\mathrm{m}}$ was determined in the following way. We selected 27 unblended iron lines (neutral and ionised) with $W_{\lambda}<25 \mathrm{~m} \AA$. For each of these lines, we made a synthesis using the abundance calculated via their $W_{\lambda}$. In such way, the macroturbulent broadening is the only free parameter in this synthesis. We found a quite large spread in $\xi_{\mathrm{m}}$ for these lines: $\xi_{\mathrm{m}}=8.0 \pm 1.2 \mathrm{~km} \mathrm{~s}^{-1}$. This is also exactly the value 
found in the Luck et al. analysis. Hence for the lithium synthesis $\xi_{\mathrm{m}}=8.0 \mathrm{~km} \mathrm{~s}^{-1}$ was used. This yielded $\log \epsilon(\mathrm{Li})=1.9$.

An error analysis on the $\mathrm{Li}$ abundance was made by varying all parameters involved in the synthesis. As expected, a temperature change has far the largest impact on the abundance: a temperature variation of $\pm 250 \mathrm{~K}$ yields a change in the $\mathrm{Li}$ abundance of $\sim 0.2$ dex. Other variations are listed in Table 4 .

\subsubsection{Europium synthesis}

The effect of hyperfine splitting ( $h f s$ ) on the europium abundance was investigated by the synthesis of the two Eu II lines of our analysis. $\log g f$ data and hyperfine splitting constants were taken from Lawler et al. (2001b). The influence of hfs was determined by integrating the profile of the synthesized line, with and without hfs decomposition. This calculated equivalent width is then compared with the measured equivalent width. In this way, the exact profile of the line is eliminated, and no broadening factors have to be applied. The effect on abundance of hfs for the resonance line at $4129.725 \AA\left(W_{\lambda}=43.1 \mathrm{~m} \AA\right)$ is significant. A hfs treatment of this line yields $\log \epsilon(\mathrm{Eu})=$ $-0.87 \mathrm{dex}$, while without hfs it is -0.79 dex. For the weak line at $6645.064 \AA\left(W_{\lambda}=5.9 \mathrm{~m} \AA\right)$, the difference between an hfs and a non-hfs treatment is not significant.

\subsection{Abundances: summary}

Referring to Fig. 3, we will now discuss the derived abundances of HD 190390.

Metallicity. With an iron abundance of $\log \epsilon(\mathrm{Fe})=5.95$, HD 190390 is clearly metal deficient by almost a factor of 40. The other iron peak elements follow this deficiency within 0.1 dex, except $\mathrm{Mn}$ and $\mathrm{Zn}$. The slight manganese deficiency $([\mathrm{Mn} / \mathrm{Fe}]=-0.3)$ is expected given the galactic chemical evolution (e.g. Nissen et al. 2000; Prochaska \& McWilliam 2000). The zinc abundance $([\mathrm{Zn} / \mathrm{Fe}]=+0.2)$ is somewhat higher than expected, but it is based on only three lines.

CNO. Although the abundance of the CNO elements is solely based on very small lines, a clear pattern for these three elements is observed: $[\mathrm{C} / \mathrm{Fe}]=-0.6$ and $[\mathrm{N} / \mathrm{Fe}]=[\mathrm{O} / \mathrm{Fe}]=+0.5$. The deficient carbon and the overabundant oxygen lead to a very low $\mathrm{C} / \mathrm{O}$ number ratio: $\mathrm{C} / \mathrm{O}=0.05$. Nitrogen seems to be enhanced at the same level as oxygen.

$\alpha$-elements. The mean of the [el/Fe] values of the $\alpha$-elements $\mathrm{Mg}, \mathrm{Si}, \mathrm{S}, \mathrm{Ca}$ and $\mathrm{Ti}$ yields $[\alpha / \mathrm{Fe}]=+0.4$. This enhancement is what is expected for stars in this metallicity range and hence the $\alpha$-elements are not intrinsically enhanced. The abundances of the individual $\alpha$-elements all agree within $0.1 \mathrm{dex}$ with this +0.4 number.

S-process elements. A very complete abundance pattern could be obtained for the s-process elements, with 53 lines of the light $(l s)$ and 83 lines of the heavy $(h s)$ s-process elements. The s-process elements are all slightly enhanced, but the abundance pattern reveals that the enhancement is probably not intrinsic. All elements are compatible with $[\mathrm{el} / \mathrm{Fe}]=0$ considering the line-to-line scatters, except $\mathrm{Zr}$, La, Eu and Hf. For Zr,
Table 4. Error analysis of the Li synthesis. A temperature change has the largest effect on the derived $\mathrm{Li}$ abundance.

\begin{tabular}{rrlr}
\hline \hline Parameter & Symbol & Variation & $\begin{array}{r}\text { Variation } \\
\log \epsilon(\mathrm{Li})\end{array}$ \\
\hline Temperature & $T_{\mathrm{eff}}$ & $\pm 250 \mathrm{~K}$ & \pm 0.20 \\
Gravity & $\log (g)$ & $\pm 0.5(\mathrm{cgs})$ & \pm 0.05 \\
Microturb. velocity & $\xi_{\mathrm{t}}$ & $\pm 2 \mathrm{~km} \mathrm{~s}^{-1}$ & \pm 0.04 \\
Macroturb. velocity & $\xi_{\mathrm{m}}$ & $\pm 2 \mathrm{~km} \mathrm{~s}^{-1}$ & \pm 0.04 \\
\hline
\end{tabular}

a large spread is seen in the $[\mathrm{Zr} / \mathrm{Fe}]$ of unevolved disk and halo stars from -0.1 to +0.6 dex (e.g. Travaglio et al. 2004). Moreover, the same relatively high $\mathrm{Zr}$ abundance is also seen in our analysis of the non-enriched RV Tauri star DS Aqr (Deroo et al. 2005). Eu is an element with a clear r-process origin, and an enhancement of $[\mathrm{Eu} / \mathrm{Fe}]=+0.4$ is certainly expected in this metallicity range (e.g. Travaglio et al. 1999). The Hf abundance is based on only one very small line and should therefore be interpreted with care. Moreover, the galactic chemical evolution of this very heavy element is still unknown. Finally, La seems to be a little bit more enhanced than the other s-process elements, but still compatible with no intrinsic enhancement regarding the scatter (although not as pronounced as for $\mathrm{Zr}$ ) for unevolved halo stars (e.g. Travaglio et al. 1999). This result is contrary to the s-process enrichment claimed by Luck et al. (1990). They found a mild enhancement by a factor of 4 compared to solar. A detailed inspection of their results, however, reveals large line-to-line scatters for all elements $(>0.25$, except for $\mathrm{Ba}$ ) and a smaller number of lines per element. Also, the abundances of the s-process elements as determined by Luck et al. do not display a consistent pattern in the sense that some elements are much more enhanced than others. $[\mathrm{Sm} / \mathrm{Fe}]=+2.1$ together with $[\mathrm{Ce} / \mathrm{Fe}]=+0.2$ is theoretically very difficult to reconcile with an s-process signature. We conclude that the s-process abundances presented here are much more reliable than the ones from the Luck et al. paper.

Lithium. The lithium abundance found in this analysis $(\log \epsilon(\mathrm{Li})=1.9)$ is 0.5 dex lower than that found in the Luck et al. analysis. The reason is the higher effective temperature in the latter analysis. Indeed, taking a model with a temperature of $6500 \mathrm{~K}$ in the Li synthesis, our derived Li abundance rises by almost 0.2 dex.

Other elements. Sodium is slightly underabundant, but not at a significant level (e.g. Carretta et al. 2000). The slight scandium enhancement $([\mathrm{Sc} / \mathrm{Fe}]=+0.1)$ is not unusual in this metallicity range (e.g. Nissen et al. 2000; Prochaska \& McWilliam 2000). Finally, the vanadium (V) abundance is quite high, but it is based on only one line.

\section{Variability analysis}

HD 190390 has been known to be variable for quite a long time. According to the SIMBAD database, the variability of this star was first mentioned by Strohmeier et al. (1965) who reported a photographic range of 0.4 mag. However, dedicated observational efforts to study this variability were made more 
Table 5. Log of the observations in the Geneva photometric system.

\begin{tabular}{rrr|crr}
\hline \hline Year & $N$ & $\begin{array}{r}\text { Time span } \\
\text { (days) }\end{array}$ & Year & $N$ & $\begin{array}{r}\text { Time span } \\
\text { (days) }\end{array}$ \\
\hline 1978 & 2 & 74 & 1985 & 52 & 163 \\
1979 & 1 & - & 1986 & 24 & 77 \\
1982 & 29 & 57 & 1987 & 6 & 13 \\
1983 & 45 & 129 & 1988 & 21 & 118 \\
1984 & 22 & 128 & & & \\
\hline
\end{tabular}

than twenty years later. Fernie (1986) published an analysis based on 32 observations in the $u v b y \beta(R I)$ system and mentions a range in the $y$ magnitude of $0.3 \mathrm{mag}$ and a possible period of 28.4 or 11.8 days. At nearly the same time, Waelkens \& Burnet (1985) presented their frequency analysis of 123 measurements in the Geneva photometric system (Rufener 1981), obtained with the Swiss photometric telescope at the European Southern Observatory. The data covered 1040 days and revealed a most significant period of 28.49 days, accounting for $80 \%$ of the total variance. The residual scatter around the fitted light curve was attributed to irregular cycle-to-cycle variations.

The frequency analysis was made using different algorithms: the Jurkevich-Stellingwerf PDM method (Stellingwerf 1978), the CLEAN algorithm (Roberts et al. 1987) and a general multifrequency least squares fitting method (Schoenaers $\&$ Cuypers 2004). In this analysis, we will give a rough estimate of this error by using the formula given by Montgomery \& O’Donoghue (1999):

$$
\sigma_{f}=\frac{\sqrt{6} \sigma_{R}}{\pi \sqrt{N} A T}
$$

with $\sigma_{R}$ the observational error, $N$ the number of measurements, $A$ the amplitude of the signal without noise, $T$ the total time span of the observations. Different forms of this formula are given in the literature. Schwarzenberg-Czerny (1991) introduced an extra parameter $D$ in the formula in order to take correlations in the noise into account. To avoid an underestimation of the calculated error, we took $T=N$. The motivation is that always $N \leq T$ for our different data sets of HD 190390, because the object is measured at most only once per night. For $A$ we took in each analysis the amplitude of the curve fitted with the found frequency. The obtained error estimate for the proposed frequencies is only an indicative number and should be interpreted with care. It is, however, useful to compare frequencies from two data sets obtained with the same or a comparable instrument.

\subsection{Geneva photometry}

The photometric data consist of 202 measurements in the Geneva photometric system obtained with the P7 photometer on the (now decommissioned) Swiss photometric telescope in La Silla from September 1982 to August 1988. In a first approach we chose not to add the new Mercator measurements to this data set for two reasons: (1) for the sake of the homogeneity of our data set and; (2) there would be a time gap of more than ten years in our data. The Mercator measurements are discussed in a separate section (Sect. 3.4). A small log of the observations is presented in Table 5. The analysis is performed on the $V$ magnitude. We looked for frequencies between 0.01 and $0.5 \mathrm{c} / \mathrm{d}$ using a frequency step of $0.00001 \mathrm{c} / \mathrm{d}$.

\subsubsection{Frequency analysis}

All frequency analysis methods show a highly significant peak near $0.035 \mathrm{c} / \mathrm{d}$, indicated by an arrow in the high resolution $\Theta$ statistic (PDM) or in the CLEAN spectrum in Fig. 4a. The results for this frequency $f_{1}$ are summarized in Table 6 . In the following, we will use for $f_{1}$ the value $0.03501( \pm 0.00001) \mathrm{c} / \mathrm{d}$ $\left(P_{1}=28.56 \mathrm{~d}\right)$. The error estimate is obtained with formula (1). $f_{1}$ accounts for $62 \%$ of the total variance. A phase diagram for this frequency is shown in Fig. 6.

After removing ("prewhitening") this first frequency from the data, we performed a second frequency analysis on the residuals. We found a new frequency very close to $f_{1}$ : $f_{1}^{\prime}=0.03535( \pm 0.00003) \mathrm{c} / \mathrm{d}$. This frequency is documented in Table 6 and Fig. 4b. Together with $f_{1}, f_{1}^{\prime}$ accounts for $72 \%$ of the total variance (an improvement of $11 \%$ ). A phase diagram for $f_{1}^{\prime}$ is shown in Fig. 6.

In the periodograms of the once prewhitened data (Fig. 4b), we note, apart from the peak at $0.03535 \mathrm{c} / \mathrm{d}\left(f_{1}^{\prime}\right)$, another peak very close to the main frequency $f_{1}$, situated at $0.03469 \mathrm{c} / \mathrm{d}$. This second peak can easily be seen in Fig. 4c, which is a zoom-in of panel (b). The peak at $0.03469 \mathrm{c} / \mathrm{d}$ is at the position $f_{1}-\left(f_{1}^{\prime}-f_{1}\right)$ and therefore it could be the third component of a frequency triplet, together with $f_{1}$ and $f_{1}^{\prime}$. A natural question is then if this frequency is also visible in an analysis of the twice prewhitened data. The answer is positive for the CLEAN spectrum, which has a maximum at $f_{1}^{\prime \prime}=0.03457( \pm 0.00005) \mathrm{c} / \mathrm{d}$. $\Theta$ is minimal for $f=0.01729 \mathrm{c} / \mathrm{d}$, which is $1 / 2 f_{1}^{\prime \prime}$ (see Fig. $4 \mathrm{~d}$ ). $f_{1}^{\prime \prime}$ accounts for an additional $6 \%$ of the variance (from $72 \%$ to $78 \%$ ). Other details can be found in Table 6. A phase diagram for this frequency is shown in Fig. 6.

A strong argument for the presence of additional frequencies is given by a simple inspection of Fig. 5. If $f_{1}^{\prime}$ and/or $f_{1}^{\prime \prime}$ are physical, then a beating should be observed with frequency $f_{\mathrm{b}}=f_{1}^{\prime}-f_{1}=0.00034 \mathrm{c} / \mathrm{d}$ or with a period of $P_{\mathrm{b}} \simeq 3000 \mathrm{~d}$. This beating can be seen in Fig. 5: the peak-to-peak amplitude per year clearly varies, reaching its maximum in 1983 (0.355 mag) and its minimum in $1986(0.099 \mathrm{mag})$. The dashed line is a simple harmonic fit with frequency $f_{\mathrm{b}}$ through the extrema (either minimum or maximum) of each year in which observations have taken place.

In the periodograms of the once prewhitened data, a prominent peak is also seen at $0.09154 \mathrm{c} / \mathrm{d}$ (Figs. $4 \mathrm{~b}$ and c). The same peak is also present in the twice prewhitened data (Fig. 4d). Since this frequency is not linked to the frequencies around $0.035 \mathrm{c} / \mathrm{d}$, we can consider this frequency as a genuine second frequency $f_{2}=0.09154( \pm 0.00005) \mathrm{c} / \mathrm{d}$. $f_{2}$ is also the highest peak in the CLEAN periodogram after removing the three frequencies around $0.035 \mathrm{c} / \mathrm{d}$. A phase diagram for this second frequency is shown in Fig. 6. 

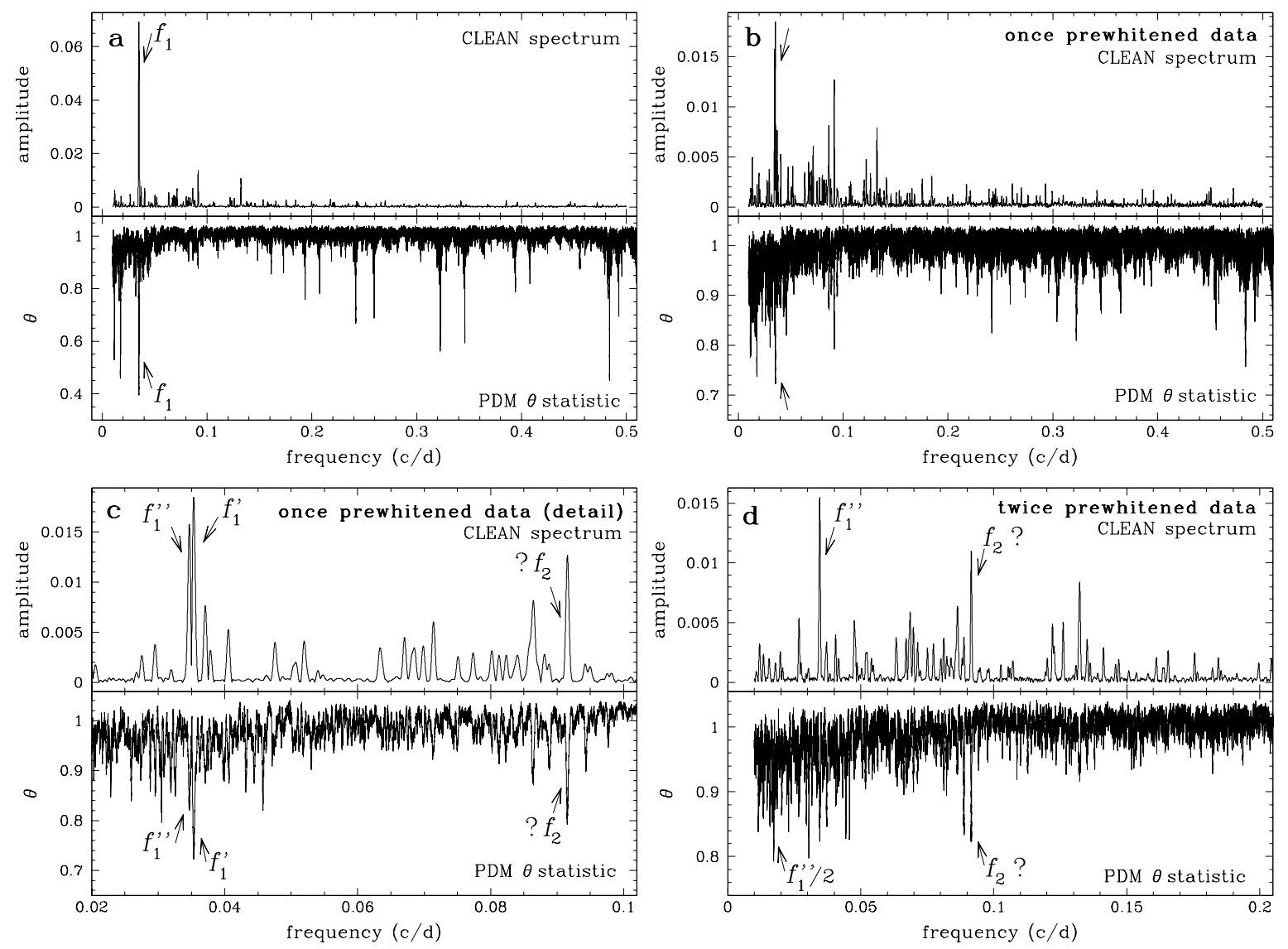

Fig. 4. The frequency analysis of the Geneva $V$ magnitude of our 202 data points. a) The high resolution $\Theta$ statistic (PDM) and the CLEAN spectrum for the first frequency $f_{1}$. Both show a highly significant peak at $0.03501 \mathrm{c} / \mathrm{d}$. b) The high resolution $\Theta$ statistic (PDM) and the CLEAN spectrum of the once prewithened data. Both show a peak at $0.03535 \mathrm{c} / \mathrm{d}$. c) Detail of the high resolution $\Theta$ statistic and the CLEAN spectrum of the once prewithened data. This figure clearly indicates the existence of a second peak $\left(f_{1}^{\prime \prime}\right)$, equidistant from $f_{1}$ compared to $f_{1}^{\prime}$. d) The high resolution $\Theta$ statistic (PDM) and the CLEAN spectrum of the twice prewithened data. The CLEAN periodogram shows a peak at $0.03457 \mathrm{c} / \mathrm{d}, \Theta$ is minimal for $0.01729 \mathrm{c} / \mathrm{d}$, which is $1 / 20.03457$.

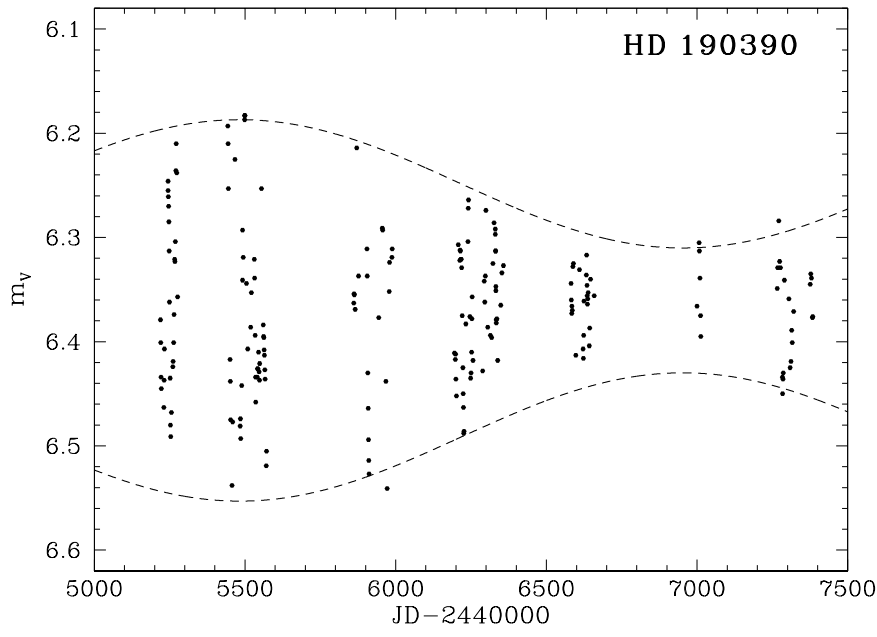

Fig. 5. The Geneva $V$ magnitude together with a harmonic fit with frequency $f_{\mathrm{b}}=f_{1}^{\prime}-f_{1}=0.00034 \mathrm{c} / \mathrm{d}$ through the extrema (either minimum or maximum) of each observational season.

\subsubsection{Light curves and colour variations}

A light curve was constructed using the four frequencies we found above. The fits were obtained using a simple least-squares procedure and the results can be seen in Fig. 7. In the upper panel of this figure, we show how the light curve behaves globally. The beating is present in the light curve, even when making use of only two frequencies. However, the amplitude of the beating seems to be underestimated. In this respect, the inclusion of the fourth frequency $\left(f_{2}\right)$ improves the global behaviour of the light curve. In the lower panel of Fig. 7, a detail of the light curve between JD 2445217 and JD 2445288 is shown.

The frequency analysis presented above was based on the $V$ magnitude because this magnitude is mostly used in this kind of analysis. In Fig. 8 the colour variations and the light variations are folded on the same period. As was noted by Waelkens $\&$ Burnet (1985), the $U-B$ and $B-V$ variations are respectively in antiphase and in phase with the light variations. This result is important in the discussion of the pulsational nature of HD 190390 (Sects. 4.2 and 4.3).

\subsection{HIPPARCOS data}

53 HIPPARCOS measurements of HD 190390 were extracted from the HIPPARCOS catalogue (ESA 1997). 43 measurements had a non-zero quality flag, but at 10 more epochs the 
Table 6. Frequencies found in the Geneva photometry.

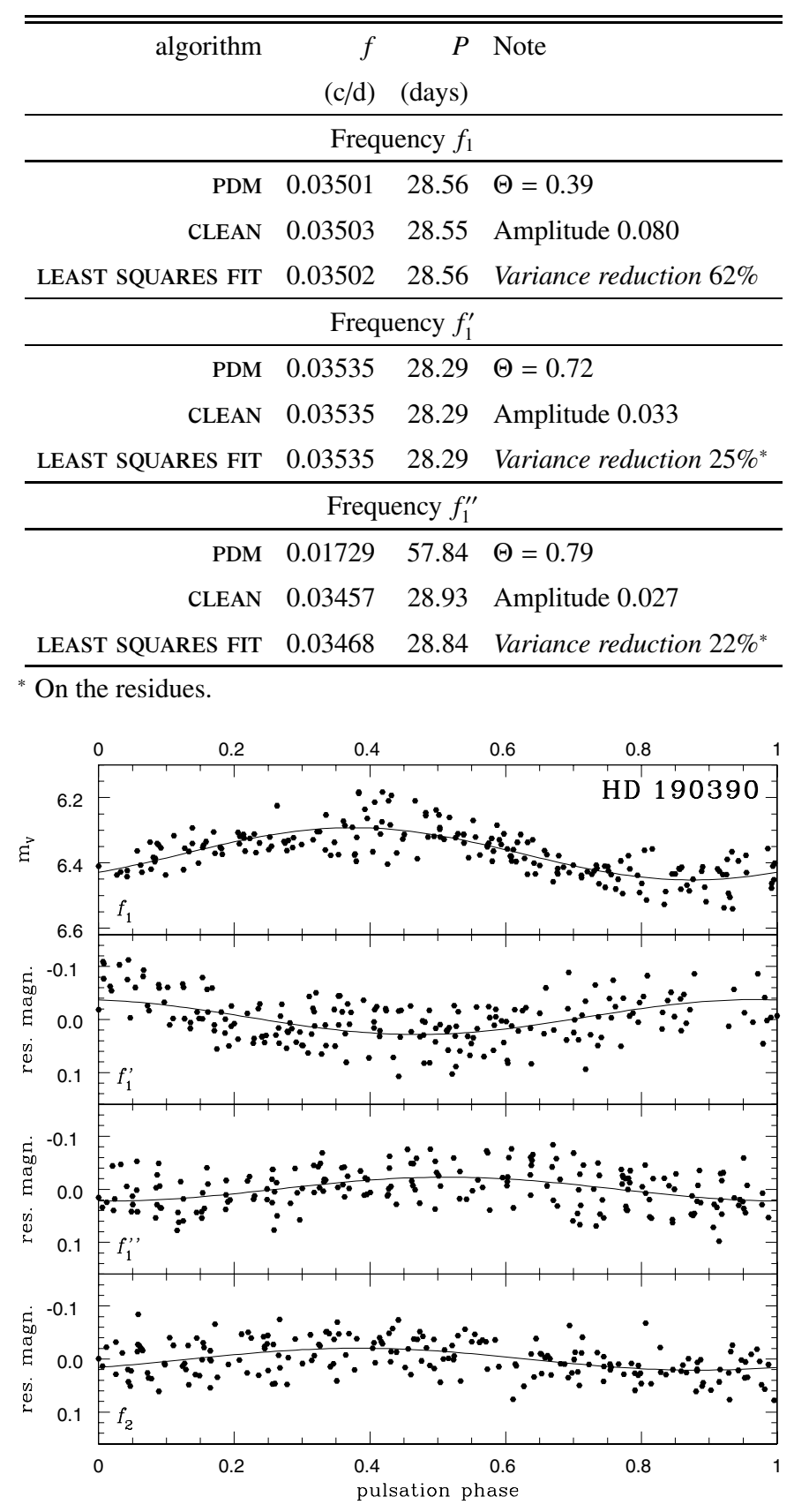

Fig. 6. Phase diagrams for the visual magnitude data of HD 190390 for the four main frequencies. Each subsequent phase diagram represents data prewhitened with the previous frequencies.

star was 0.10 to 0.15 mag brighter than on average. We have no explanation for this brightening, but since we cannot exclude that these brightenings are instrumental, we used only the remaining 34 points. They cover a total time span of 948.6 days, from April 1990 to November 1992. The precision of these HIPPARCOS magnitudes (which is also given in the catalogue for each measurement individually) is between 0.005 and $0.01 \mathrm{mag}$.

It is remarkable that also in this very limited data set, the second best two-frequency solution is the combination
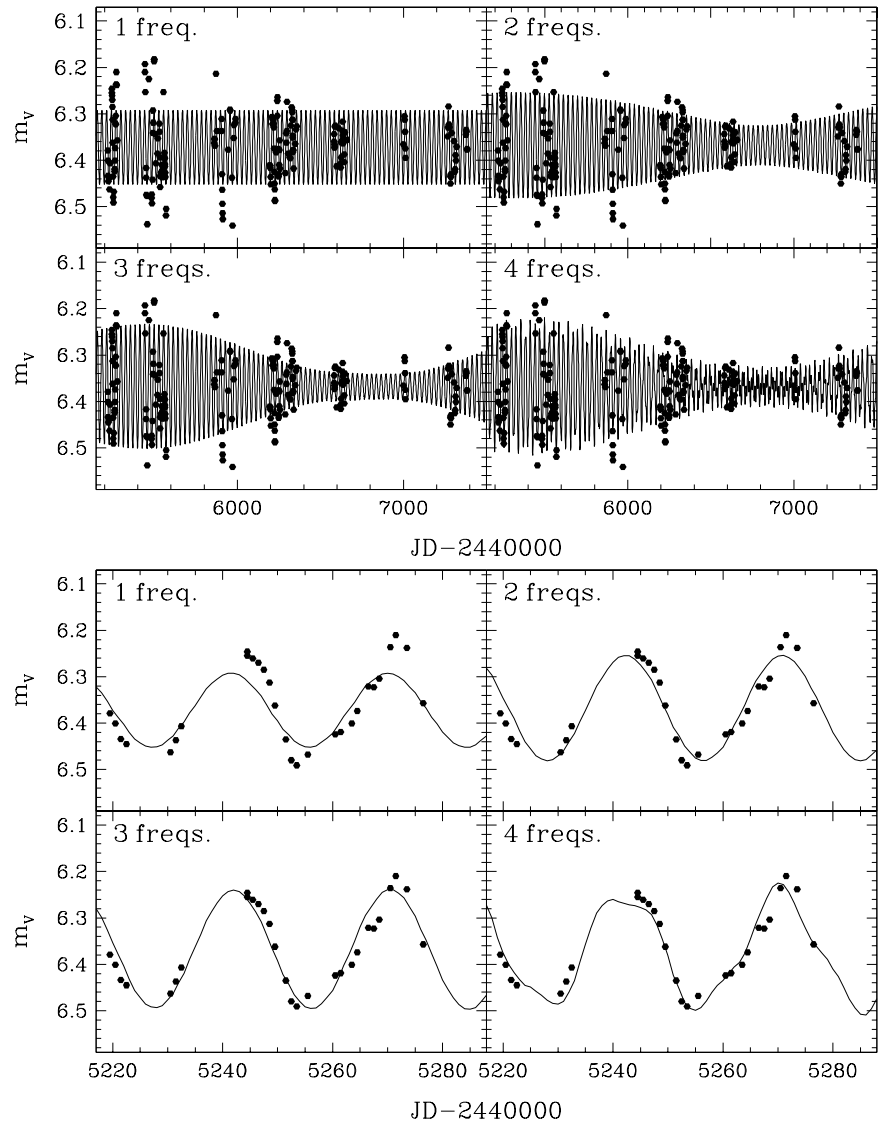

Fig. 7. Light curve of HD 190390. The light curve is constructed using the number of frequencies indicated in the upper left corner of each panel on the figure. Upper panel: global behaviour of the light curve. It is clear that the beating which was already noted in Fig. 5 is sampled quite well when making use of more than one frequency. On the other hand, the overall amplitude of the beating seems to be underestimated. Lower panel: local behaviour of the light curve of HD 190390.

of $f_{\mathrm{H} 1}=0.03704 \mathrm{c} / \mathrm{d}\left(P_{\mathrm{H} 1}=27.00 \mathrm{~d}\right)$ and $f_{\mathrm{H} 2}=0.09042 \mathrm{c} / \mathrm{d}$ $\left(P_{\mathrm{H} 2}=11.06 \mathrm{~d}\right)$. These frequencies account for $95.8 \%$ of the total variance. The best combination in the least-squares sense, $f_{\mathrm{H}^{\prime}}=0.07738 \mathrm{c} / \mathrm{d}\left(P_{\mathrm{H} 1^{\prime}}=12.92 \mathrm{~d}\right)$ and $f_{\mathrm{H} 2^{\prime}}=0.09044 \mathrm{c} / \mathrm{d}$ $\left(P_{\mathrm{H} 2^{\prime}}=11.06 \mathrm{~d}\right)$ does not give a significantly different result (96.4\% variance reduction). The frequencies $f_{\mathrm{H} 1}$ and $f_{\mathrm{H} 1^{\prime}}$ could be related aliases since the second one is not recovered after prewhitening with the first frequency and vice-versa, and there is no doubt that the star has a main period of around 28 days.

\subsection{CORALIE radial velocities}

The CORALIE fiber-fed echelle spectrograph at the Swiss $1.2 \mathrm{~m}$ telescope at La Silla has a resolution of $\sim 50000$ at $5000 \AA$ and a wavelength coverage from 3880 to $6810 \AA$. Due to the stability of the whole configuration and the possibility of making simultaneously Thorium-Argon calibration spectra in the second fibre, the spectrograph is specifically designed for high-precision radial-velocity measurements.

Since November 1999, 103 radial velocity measurements of HD 190390 have been collected (Fig. 9). Velocities are deduced by cross-correlating the spectra with an appropriate mask. 


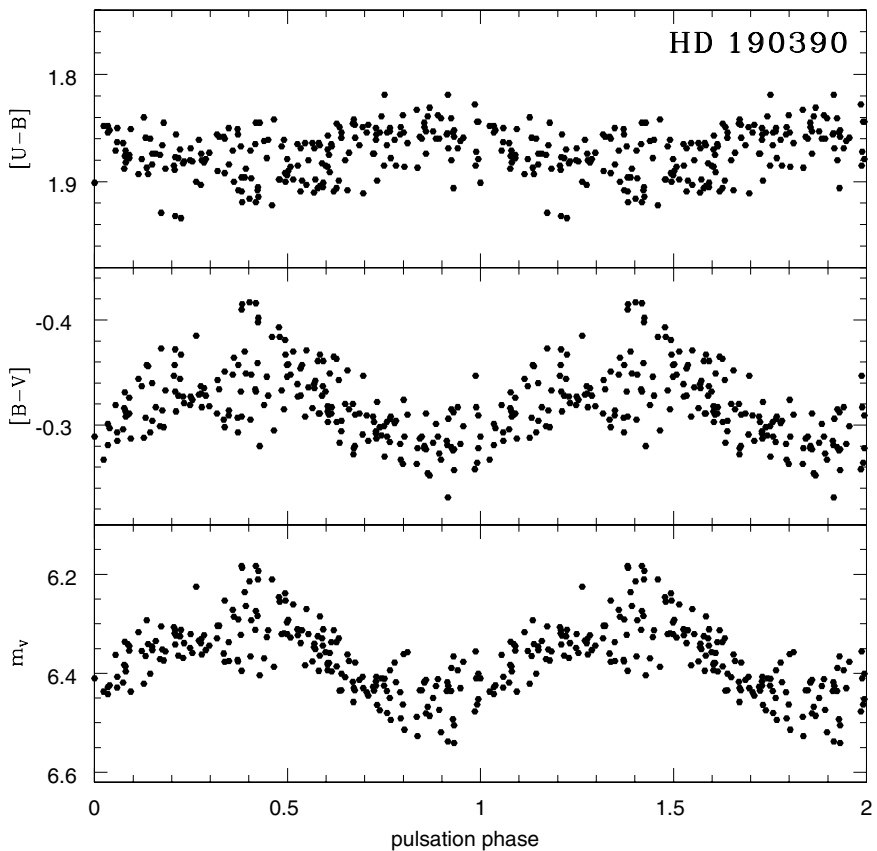

Fig. 8. The $U-B$ and $B-V$ colour variations folded on the same period $(28.56 \mathrm{~d})$ as the light variations $\left(m_{V}\right)$. The displayed colour variations are clearly in antiphase respectively phase with the light variations.

Table 7. Frequency analysis of the CORALIE radial velocity data. Note that both PDM and the LEAST SQUARES method find $1 / 2 f_{1}$ instead of $f_{1}$.

\begin{tabular}{rrrl}
\hline \hline Algorithm & $\begin{array}{r}f_{\mathrm{v}} \\
(\mathrm{c} / \mathrm{d})\end{array}$ & $\begin{array}{r}P_{v} \\
(\text { days })\end{array}$ & \\
& note \\
\hline PDM & 0.01745 & 57.31 & $\Theta=0.23$ \\
CLEAN & 0.03502 & 28.56 & Amplitude 0.93 \\
LEAST SQUARES FIT & 0.01741 & 57.44 & Variance reduction 76\% \\
\hline
\end{tabular}

In the case of HD 190390, an F0 mask is used. Sample correlation profiles for one observational run are given at the bottom of Fig. 9. The accuracy of these velocities is $\sim 1.0-1.5 \mathrm{~km} \mathrm{~s}^{-1}$. From Fig. 9 it is clear that the period in the radial velocity data must be close to the main period $f_{1}$ found in the photometric data. The frequency found in the frequency analysis (Table 7 and Fig. 10) is indeed equal to the main frequency found in the Geneva photometry.

Variation of the $\mathrm{H} \alpha$ profile during a pulsational cycle is often used as a powerful diagnostic tool in the determination of the pulsation character. The CORALIE spectra, however, are of too low signal-to-noise $(\mathrm{s} / \mathrm{n})$ for a detailed profile variation study, because the first intention of these spectra was only to obtain a radial velocity by cross-correlation. For this purpose, a s/n of around 30 is sufficient, and the mean $\mathrm{s} / \mathrm{n}$ of all spectra is indeed 40 . Nontheless, we can study line profile variations over a cycle by combining spectra in the same phase bin. In such a procedure, a limited number of higher $\mathrm{s} / \mathrm{n}$ spectra is compiled out of spectra situated in the same phase bin. In the construction of these combined spectra, we chose to use 6 phase bins designated A to F, illustrated in Fig. 11. The number of spectra
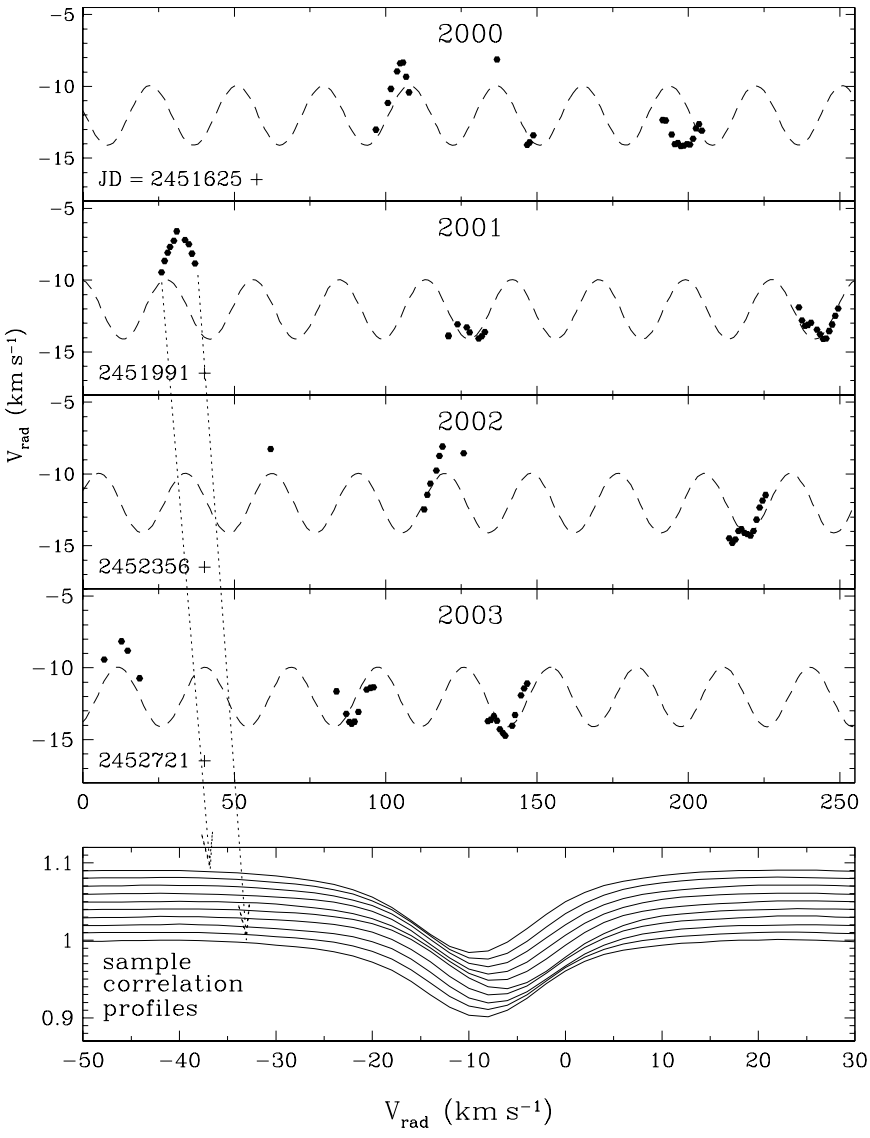

Fig. 9. The CORALIE radial velocity data from 2000 to 2003. Several groups of points can be discerned, indicating the different observational runs. A radial velocity curve with a period of $P=28.56$ days is fitted to the data. This period was found in the photometric data, and also in the CLEAN analysis of the radial velocity data. At the bottom, sample correlation profiles are given for one observational run.

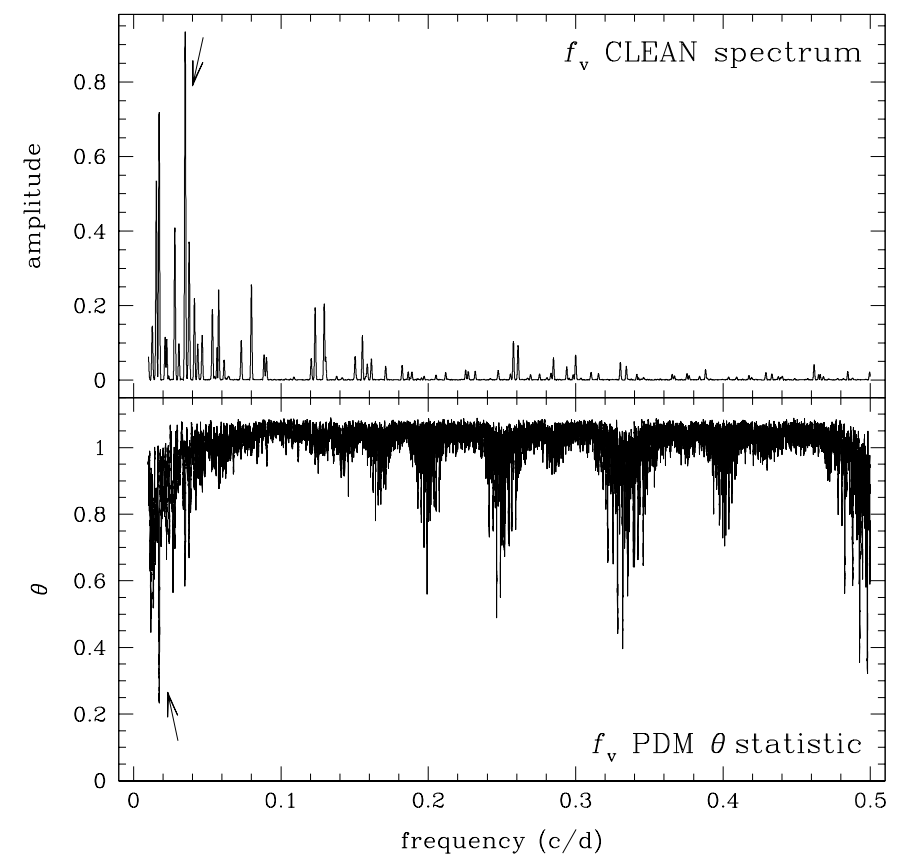

Fig. 10. The high resolution $\Theta$ statistic (PDM) and the CLEAN spectrum of the CORALIE radial velocities. CLEAN shows a peak at $0.03502 \mathrm{c} / \mathrm{d}$, while the PDM $\Theta$ statistic is minimal for $f=0.01745 \mathrm{c} / \mathrm{d}$. 

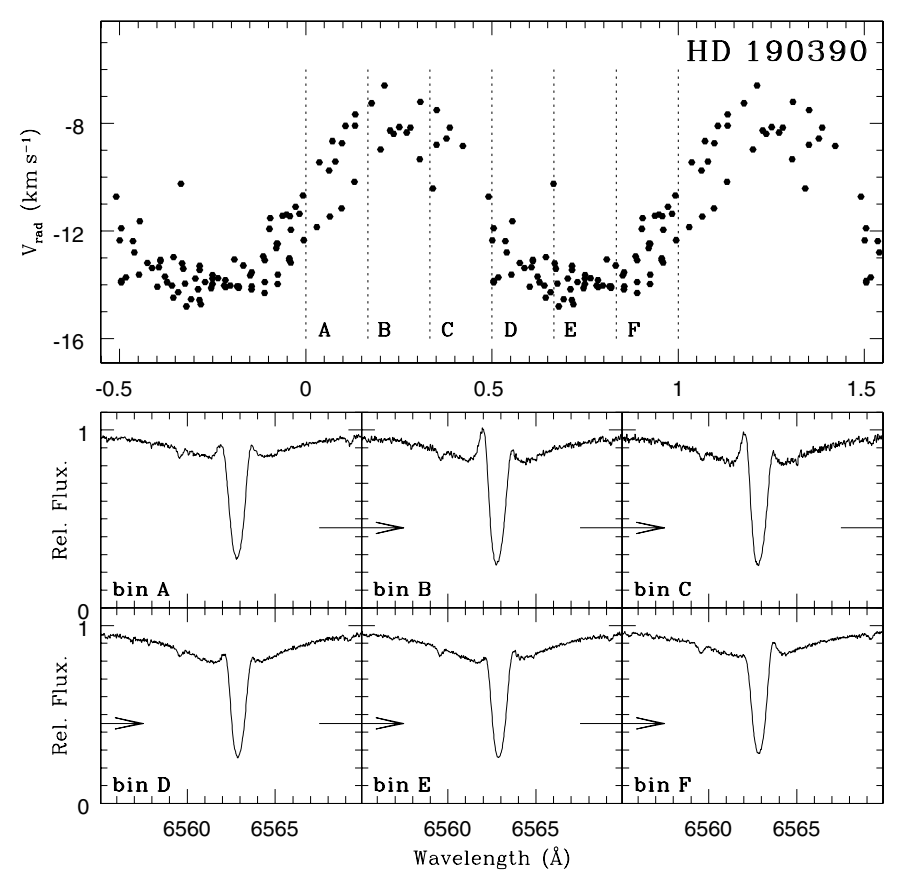

Fig. 11. Upper panel: Phase bins used in the co-adding of the 103 CORALIE spectra. In this "optimized" phase diagram with frequency $0.03501 \mathrm{c} / \mathrm{d}$, a specific phase was determined for each observing run separately. Lower panel: The $\mathrm{H} \alpha$ line in the six resulting spectra showing a different profile during one cycle.

Table 8. Details of the phase binning of the 102 CORALIE spectra into 6 bins. The older measurement of 1999 was not used.

\begin{tabular}{rrrrr}
\hline \hline & Phase bin & \# spectra & $\begin{array}{r}\text { Total integra- } \\
\text { tion time (s) }\end{array}$ & $\begin{array}{r}\text { Achieved s/n } \\
\text { (at 6550 ̊) }\end{array}$ \\
\hline A & $-0.083-0.083$ & 14 & 2968 & 160 \\
B & $0.083-0.250$ & 10 & 1713 & 100 \\
C & $0.250-0.417$ & 6 & 1200 & 90 \\
D & $0.417-0.583$ & 22 & 5632 & 150 \\
E & $0.583-0.750$ & 25 & 7343 & 180 \\
F & $0.750-0.917$ & 25 & 7172 & 150 \\
\hline
\end{tabular}

added, the total integration time and the achieved $\mathrm{s} / \mathrm{n}$ ratios are summarized in Table 8. Spectra were first transformed to rest wavelength, normalised and then added, s/n weighted.

From Fig. 11 it is clear that $\mathrm{H} \alpha$ in HD 190390 is variable during a pulsational cycle: relatively strong emission in the blue wing is seen in bins $\mathrm{B}$ and $\mathrm{C}$, while in bin $\mathrm{F}$ for example, there is only some emission present in the red wing, and none in the blue. $\mathrm{H} \alpha$ emission in the blue wing was already noted by Sasselov (1985), and later confirmed by Luck et al. (1990). Sasselov gave a $\sim 60 \mathrm{~km} \mathrm{~s}^{-1}$ separation between the emission and the photospheric rest velocity of the $\mathrm{H} \alpha$ absorption. We find similar values for this separation, but the exact value for each phase binned spectrum depends on the method adopted to determine the central wavelength of both the emission and the absorption peak.

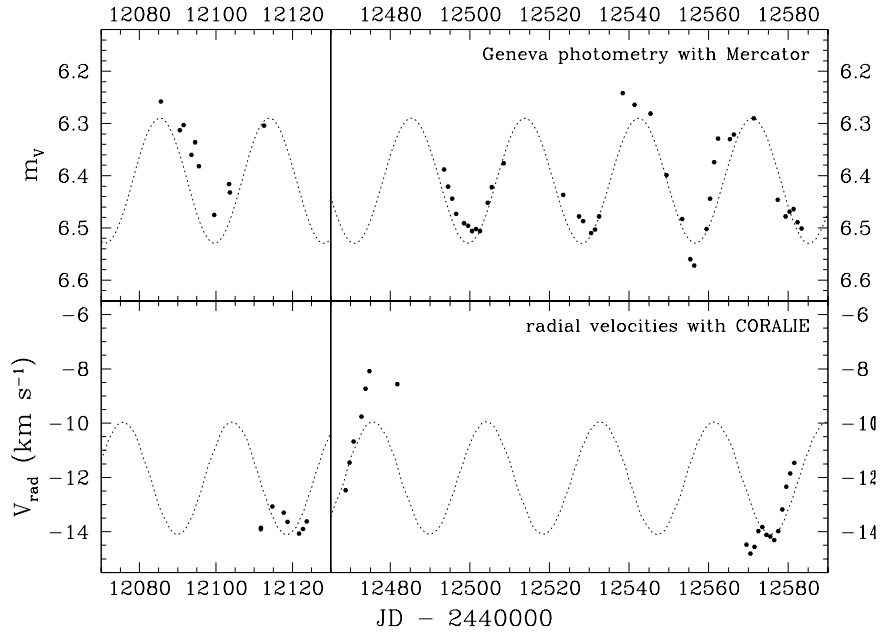

Fig. 12. Upper panel: Overview of the Geneva photometry collected with the Mercator telescope, fitted with a harmonic with the main frequency $(0.03501 \mathrm{c} / \mathrm{d})$. In the lower panel a comparison is made with the CORALIE radial velocities of the same period. The dotted line is again a harmonic with the main frequency. It is clear that both curves are mirror images from each other.

\subsection{Geneva photometry with Mercator}

The Mercator telescope is a $1.2 \mathrm{~m}$ telescope located on the Roque de los Muchachos observatory on La Palma, Spain. The Mercator telescope, operated by our Institute, is a twin of the Swiss telescope in La Silla. Two instruments are available for the moment: the refurbished photometer P7 which was in use at the "old" Swiss telescope at La Silla, and a CCD camera.

HD 190390 is in the standard program catalogue for P7, but since the declination of this object is rather low $\left(-11^{\circ} 36^{\mathrm{m}}\right)$, the minimum air mass for La Palma is quite high $\left(F_{z}=1.30\right)$. Therefore, HD 190390 is always measured in concatenation with the standard star HD 190172 (F4III, $V=6.72$ ) and the comparison star HD 192771 (G5, $V=8.7)$. 72 measurements of HD 190390 have been collected with the Mercator telescope. During the reduction, a quality flag is assigned to each observation, ranging from 0 to 4 . Observations with the lowest quality (flag 0) were discarded, and 66 measurements remained, covering 787 days. This data set is still quite limited to perform a reliable frequency analysis. However, a preliminary analysis again suggests a frequency close to the main frequency found in the "old" Geneva data: $f_{\mathrm{M}}=0.03524 \mathrm{c} / \mathrm{d}( \pm 0.00005) \mathrm{c} / \mathrm{d}$ $\left(P_{\mathrm{M}}=28.38\right.$ days $)$. This frequency accounts for $86 \%$ of the total variance.

There is a secondary peak in the periodograms near the main frequency and at $0.0897 \mathrm{c} / \mathrm{d}$ an unrelated frequency is found. The best 3 -frequency solution ( $94 \%$ reduction of the initial variance) is with $0.03521,0.04072$ and $0.08970 \mathrm{c} / \mathrm{d}$.

\subsection{Variability}

\subsubsection{Photometry and velocity}

In Fig. 12 we compare the light curve of HD 190390 with its radial velocity curve. The comparison is made for a time interval for which we have both photometric and velocity data, so 


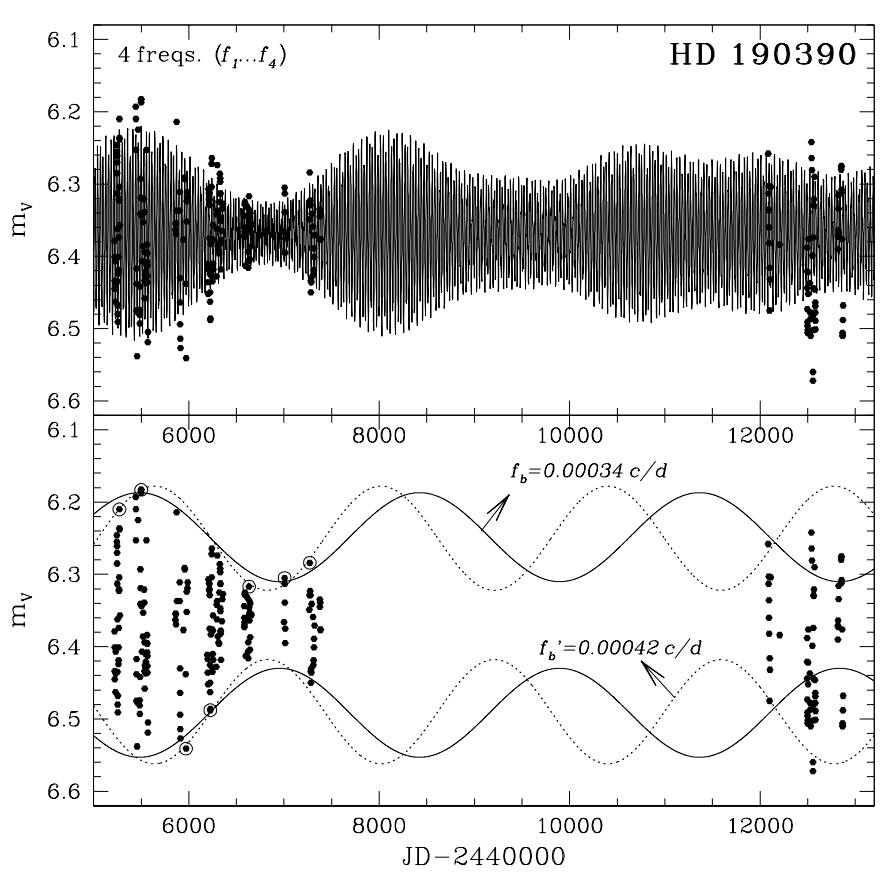

Fig. 13. Comparison of the new Mercator photometry and the "old" Geneva photometry. In the upper panel the light curve of HD 190390 constructed with four frequencies is extrapolated to the time range covered by the Mercator data (points right on the figure). Extrapolation of the light curve is, however, far from robust, since a small change in frequency will cause a rather large change in the global behaviour of the light curve in the extrapolated region. In the lower panel a harmonic fit with frequency $f_{\mathrm{b}}=f_{2}-f_{1}=0.00034 \mathrm{c} / \mathrm{d}$ (full line) and frequency $f_{\mathrm{b}}^{\prime}=0.00042 \mathrm{c} / \mathrm{d}$ (dotted line) through the extrema (marked on the figure with an open dot) of each year (1982-1988) is constructed. The latter frequency is the best fit through the extrema, while the former was found in our frequency analysis (see Table 6).

no extrapolation is needed. Both curves are fitted with a simple harmonic with the main frequency $(0.03501 \mathrm{c} / \mathrm{d})$. It is clear that both curves are mirror images from each other.

\subsubsection{Beating in the Mercator data}

To determine whether the beating is seen in the Mercator data, we extended the light curve constructed in Fig. 7 to the time range covered by the Mercator data (upper panel of Fig. 13). We remind that the amplitude of the light curve seems to be underestimated. Thus, the points of the second observational run on Mercator being quite off the light curve (by $\sim 0.12 \mathrm{mag}$ ) is not an argument to reject a stable beating. Caution is needed because a small difference in the frequencies will cause a quite large difference in the global behaviour of the extrapolation.

Another and more robust method is depicted in the lower panel of the same figure. There, a simple harmonic fit was constructed through the extreme values (either minimum or maximum) of each year (1982-1988) and with a fixed frequency of $f_{\mathrm{b}}=f_{1}^{\prime}-f_{1}=0.00034 \mathrm{c} / \mathrm{d}$. This fit is then mirrored with respect to the mean $m_{V}$ value, and an enveloping curve is obtained. The data points of the second Mercator run again fall out of the permitted region, so they do not seem to be compatible with the beating. However, with a small change in the beating frequency $\left(f_{\mathrm{b}}^{\prime}=0.00042 \mathrm{c} / \mathrm{d}\right)$, we were able to make these points "permitted".

Thus, a beating phenomenon is not excluded although the values of the frequencies around the main frequency seem not well defined. If a very close duplet or triplet of frequencies is present, the total time span of the observations could still be insufficient to resolve the exact frequency values. Alternatively, it is not excluded that the main frequency $f_{1}$ changes its value and its amplitude in time in a more or less random way. In this case the nearby frequencies are only "remnants" of the broadened peak in the periodogram not dealt with by the prewhitening.

For the secondary frequency, near $0.09 \mathrm{c} / \mathrm{d}$, we found indications of its presence in independent data sets, and it might correspond to the frequency $f=0.0847 \mathrm{c} / \mathrm{d}$ that was already found by Fernie (1986). Here also its value is either variable in time or some beating of closely spaced, so-far unresolved frequencies is present.

\section{Discussion}

\subsection{Luminosity}

In this first section of the discussion, the luminosity of HD 190390 is estimated. As a first step, the reddening of the object is quantified by calculating synthetic colour indices. Then, two different methods are used to obtain the luminosity of our object.

Synthetic colour indices and reddening. The reddening is estimated by comparing the observed Geneva colours of our object with calculated synthetic colours. Such synthetic colours were calculated by combining the digitized passband of the Geneva filters (Rufener \& Nicolet 1988) with the Kurucz model atmospheres (see Reyniers 2002, for more details). The synthetic $B-V$ of HD 190390 with model $\left(T_{\text {eff }}, \log g,[\mathrm{M} / \mathrm{H}]\right)=$ $(6250 \mathrm{~K}, 1.0,-1.5)$ is $B-V=-0.574$. The mean $B-V$ in our Geneva data is $B-V=-0.318$, hence $E(B-V)=$ $0.256 \pm 0.05 \mathrm{mag}$. This value is somewhat higher than the value derived by Fernie (1986): $E(B-V)=0.10 \mathrm{mag}$.

Luminosity with trigonometric parallax. The parallax found in the HIPPARCOS catalogue (retrieved via SIMBAD) is $\pi=$ $2.56( \pm 0.97)$ mas, which gives a distance of $d=391 \mathrm{pc}$, with an upper limit of $629 \mathrm{pc}$ and a lower limit of $283 \mathrm{pc}$. Taking the reddening into account, we get an absolute visual magnitude of $M_{V}=-2.29$. However, the uncertainty induced by the parallax is much larger than the uncertainty of the reddening:

$d=629 \mathrm{pc} \rightarrow M_{V}=-3.33$

$d=283 \mathrm{pc} \rightarrow M_{V}=-1.60$.

Assuming the bolometric correction to be negligible (see e.g. Flower 1996), we derive a luminosity of $L=656 L_{\odot}$. An upper limit is obtained for this method by taking $d=629 \mathrm{pc}$, which yields $L=1701 L_{\odot}$ or $\log \left(L / L_{\odot}\right)=3.2$.

Luminosity with $\boldsymbol{P}-\boldsymbol{L}-[\mathbf{F e} / \mathbf{H}]$ relation. Nemec et al. (1994) derived P-L- $[\mathrm{Fe} / \mathrm{H}]$ relations and distances for several classes of Pop. II variables. Given the pulsational properties of HD 190390 together with its metal deficiency, there is 
strong evidence that this object belongs to the class of Pop. II Cepheids (see also Sect. 4.3). The P-L-[Fe/H] relation for this class depends on the pulsation mode (either fundamental or first-overtone). With the P-L- $[\mathrm{Fe} / \mathrm{H}]$ relations given in Nemec et al. (1994), and $P=28.56$ d, we obtain

$$
\begin{array}{ll}
M_{V}(\mathrm{~F})=-2.47( \pm 0.07) & \text { (fundamental) } \\
M_{V}(\mathrm{H})=-2.92( \pm 0.07) & \text { (first overtone) }
\end{array}
$$

where the error of 0.07 only accounts for the error on the $\mathrm{P}-\mathrm{L}-[\mathrm{Fe} / \mathrm{H}]$ relation. This error is, again, probably underestimated, not only because the variables in the formula are uncertain (especially $[\mathrm{Fe} / \mathrm{H}]$ ), but also because the relation was derived with data from cluster variables. It is therefore not clear if this relation also holds for field stars like HD 190390. Note also that with a maximum magnitude of $M_{V}=-3$, we derive a distance of $d=673 \mathrm{pc}\left(\log \left(L / L_{\odot}\right)=3.1\right.$ and distance from the galactic plane $|Z|=247 \mathrm{pc})$.

\subsection{UU Her classification}

UU Her type stars are defined by Sasselov (1984) as variable F-type supergiants at high galactic latitudes having long periods and low amplitudes. Further characteristics of this group are (Bartkevičius 1992):

- metal deficiency;

- high velocities;

- very specific variability with relatively small amplitudes and two or three alternating periods form 20 to 120 days;

- large infrared (IR) excesses due to circumstellar dust.

However, an object does not have to possess all properties mentioned here to be a member of this heterogeneous class. The evolutionary status of these stars is not yet clear, but most characteristics clearly point to a final stage of high luminosity or a post-AGB stage.

Given its low metal content already mentioned by McDonald (1976) and its high galactic latitude, HD 190390 was mentioned as a possible UU Her candidate (Waelkens \& Burnet 1985; Sasselov 1985; Fernie 1986). However, due to the apparently stable and short period (relative to other UU Her candidates) found by Waelkens \& Burnet, the genuine character of HD 190390 as UU Her star was doubted. Moreover, HD 190390 has a "normal" radial velocity and no significant infrared excess has been observed by IRAS.

With the observational material presented here, we are able to further contest this UU Her classification. We confirm the stable Waelkens \& Burnet period of $\sim 28.6 \mathrm{~d}$. Our radial velocity data further confirm the "normal" velocity of HD 190390. Also the fact that there is no significant reddening is opposite to what is seen in most UU Her type stars. Therefore, a post-AGB stage of our object is less likely. Also,

- the chemical signature does not point to a 3rd dredge-up scenario (no carbon or s-process enhancement);

- the (however very uncertain) luminosity (not higher than $\log L / L_{\odot} \simeq 3.2$ ) is too low to support a post-AGB stage.

The absence of a clear 3rd dredge-up signature is, however, not enough to exclude a post-AGB scenario (e.g. Van Winckel 1997).

\subsection{W Vir classification}

W Virginis variables are pulsating stars of population II located in the Cepheid instability strip. In the HR diagram, W Vir stars occupy a region slightly lower and slightly to the right compared to the classical (Pop. I) Cepheids. Periods are between 12 and about 20 days and amplitudes between 0.3 and 1.2 mag. W Vir stars are in fact part of the larger class of Pop. II Cepheids, containing also the BL Her stars at the shorter period end $(P=1-5 \mathrm{~d})$, and the RV Tauri stars at the longer period end $(P=20-100 \mathrm{~d})$; see Wallerstein (2002) for a review of Pop. II Cepheids. Spectra of W Vir stars sometimes show hydrogen and helium emission near maximum light, and double metallic absorption lines, usually explained by the propagation of a shock wave through thephotosphere.

The metal deficiency, the luminosity (too low for post-AGB) and most importantly the length of the period itself suggest a W Vir classification for HD 190390. Furthermore, the CNO chemical pattern points to a 1 st (post-RGB) rather than a 3rd (post-AGB) dredge up. The (anti-)correlations seen in the colours are reminiscent of variables in the instability strip. Moreover, the luminosity inherent to this classification is compatible (within the error) with the luminosity derived by the trigonometric parallax. On the other hand, the spectroscopic features seen in W Vir (strong hydrogen and helium emission and double metallic absorption lines) are not seen in the spectrum (except the weak emission in the wing of $\mathrm{H} \alpha$ ). The photosphere of HD 190390 is seemingly much more stable than that of W Vir, and no strong shock wave is developed during the pulsation.

\subsection{Lithium in HD 190390}

In Sect. 2.3 we derived a $\mathrm{Li}$ abundance of $\log \epsilon(\mathrm{Li})=1.9$. For an extensive discussion of $\mathrm{Li}$ in evolved stars, we refer to Sect. 5.2 in Reyniers \& Van Winckel (2001). The Li abundance of HD 190390 is not higher than the interstellar Li abundance of $\log \epsilon(\mathrm{Li})=3.1$. However, since the initial $\mathrm{Li}$ abundance of HD 190390 (thought to be the Spite plateau) is approximately the present one, and since at least one mixing event has occurred in HD 190390 (as seen from the CNO pattern), the Li content is expected to be much lower than the initial one. Note that a Li dilution of a factor of 20 during the 1st dredge-up is expected for stars in this metallicity range (e.g. Gratton et al. 2000). Therefore, Li production is required in the case of HD 190390.

An obvious Li producing scenario for HD 190390, which was also invoked by Luck et al. (1990), is a "hot bottom burning" (HBB) scenario in which the base of the convective envelope of a AGB star is hot enough for the hydrogen burning to ignite and in which fresh ${ }^{7} \mathrm{Li}$ is quickly transported to cooler layers before it is destroyed by proton capture. However, only AGB stars of intermediate mass (4-5 $M_{\odot}$ ) or equivalently high luminosity $M_{\text {bol }} \lesssim-6$ are thought to exhibit this HBB, and therefore such a scenario is difficult to reconcile with a W Vir or post-AGB classification. There exists also a class of Li enriched galactic low mass field Carbon stars (Abia et al. 1993; 
Abia \& Isern 1997, 2000), but these stars are chemically very different from HD 190390.

Turning to less evolved stars, $\mathrm{Li}$ enrichment is also seen in about one percent of "normal" G-K giants. Some of these giants show Li abundances even larger than that of the interstellar medium. While the exact mechanism responsible for this enrichment is still not known, internal production of ${ }^{7} \mathrm{Li}$ is also for this group of stars most favourable (e.g. de La Reza et al. 1997). Non-standard mixing models are developed to explain these high $\mathrm{Li}$ abundances and commonly dubbed "cool bottom processing" (CBP, Wasserburg et al. 1995; Sackmann \& Boothroyd 1999). An attractive but highly speculative scenario for the Li enhancement in HD 190390 could be a scenario in which HD 190390 turned into a Li-rich K giant during its ascent on the red giant branch and in which it was able to preserve this $\mathrm{Li}$ in its outer layers.

The 25.7 day Population II Cepheid V42 in the globular cluster M 5 (Carney et al. 1998) is a metal poor $([\mathrm{Fe} / \mathrm{H}]=$ $-1.22)$ star with a surprisingly high $\mathrm{Li}$ abundance of $\log \epsilon(\mathrm{Li}) \simeq$ 1.8. No intrinsic neutron-capture element enhancement was observed either. Using the distance to M 5, the authors give an estimate for the mean absolute visual magnitude of $M_{V}=-3.15$. Apart from these characteristics very similar to HD 190390, some differences are also seen. Oxygen for example is slightly deficient in this star, whereas sodium is enhanced. This could be important evidence that deep mixing has taken place, in which products of the $\mathrm{ON}$ part of the $\mathrm{CNO}$ cycle are brought from the hydrogen-burning shell to the surface.

\section{Conclusion}

In this paper an attempt was made to clarify the evolutionary status of the luminous F-type supergiant HD 190390. The star was previously classified as a post-AGB star, based on its high galactic latitude and metal deficiency. However, no unambiguous chemical indications were found (carbon and s-process enrichment) to support this view.

Here, we confirm the main pulsational period of $\sim 28.6 \mathrm{~d}$. The high resolution, high signal-to-noise spectra confirmed the metal deficiency, the $\mathrm{C}$ deficiency, the $\mathrm{N}$ and $\mathrm{O}$ enhancement and the unexpectedly high $\mathrm{Li}$ abundance. A detailed abundance study of the s-process elements indicated a slight s-process enhancement which is in our view probably not intrinsic, but a consequence of the (small) scatter in the galactic chemical evolution. We were not able to give a conclusive evolutionary status for this star, but contrary to the post-AGB classification found in the literature, we suggest a W Vir classification instead.

Analysis of the different independent data sets showed that in this star a second periodicity is present near $11.1 \mathrm{~d}$. We suggest that the pulsation has a beating with frequency $f_{b}=0.00034 \mathrm{c} / \mathrm{d}$. This could be a working hypothesis guiding future observations of HD 190390.

Acknowledgements. M.R. acknowledge financial support from the Fund for Scientific Research - Flanders (Belgium). This research has made use of the Vienna Atomic Line Database (VALD), operated at Vienna, Austria, and the SIMBAD database, operated at CDS, Strasbourg, France. Hans Van Winckel, Peter De Cat and Katrien Kolenberg are warmly thanked for many improvements on early versions of the manuscript.

\section{References}

Abia, C., Boffin, H. M. J., Isern, J., \& Rebolo, R. 1993, A\&A, 272, 455

Abia, C., \& Isern, J. 1997, MNRAS, 289, L11

Abia, C., \& Isern, J. 2000, ApJ, 536, 438

Bartkevičius, A. 1992, Baltic Astron., 1, 194

Biémont, E., Hibbert, A., Godefroid, M., \& Vaeck, N. 1993, ApJ, 412, 431

Biémont, E., Hibbert, A., Godefroid, M., Vaeck, N., \& Fawcett, B. C. 1991, ApJ, 375, 818

Bidelman, W. P. 1951, ApJ, 113, 304

Blackwell, D. E., Shallis, M. J., \& Simmons, G. J. 1980, A\&A, 81, 340

Carney, B. W., Fry, A. M., \& Gonzalez, G. 1998, AJ, 116, 2984

Carretta, E., Gratton, R. G., \& Sneden, C. 2000, A\&A, 356, 238

de La Reza, R., Drake, N. A., da Silva, L., Torres, C. A. O., \& Martin, E. L. 1997, ApJ, 482, L77

Deroo, P., Reyniers, M., Van Winckel, H., Goriely, S., \& Siess, L. 2005, A\&A, submitted

ESA. 1997, The Hipparcos and Tycho Catalogues, ESA SP-1200

Fernie, J. D. 1986, ApJ, 301, 302

Flower, P. J. 1996, ApJ, 469, 355

Giridhar, S., Ferro, A., \& Parrao, L. 1997, PASP, 109, 1077

Gratton, R. G., Sneden, C., Carretta, E., \& Bragaglia, A. 2000, A\&A, 354, 169

Grevesse, N., \& Sauval, A. J. 1998, Space Science Rev., 85, 161

Hibbert, A., Biémont, E., Godefroid, M., \& Vaeck, N. 1991, A\&AS, 88,505

Holweger, H. 2001, in Joint SOHO/ACE workshop, Solar and Galactic Composition, AIP Conf. Proc., 598, 23

Kupka, F., Piskunov, N., Ryabchikova, T. A., Stempels, H. C., \& Weiss, W. W. 1999, A\&AS, 138, 119

Kurucz, R. 1993, CD-ROM set, Cambridge, MA: Smithsonian Astrophysical Observatory

Lambert, D. L., Heath, J. E., Lemke, M., \& Drake, J. 1996, ApJS, 103, 183

Lawler, J. E., Bonvallet, G., \& Sneden, C. 2001a, ApJ, 556, 452

Lawler, J. E., Wickliffe, M. E., Den Hartog, E. A., \& Sneden, C. 2001b, ApJ, 563, 1075

Luck, R. E., Bond, H. E., \& Lambert, D. L. 1990, ApJ, 357, 188

McDonald, L. 1976, BAAS, 8, 49

Montgomery, M. H., \& O’Donoghue, D. 1999, Delta Scuti Newsletter, 13,28

Nemec, J. M., Nemec, A. F. L., \& Lutz, T. E. 1994, AJ, 108, 222

Nissen, P. E., Chen, Y. Q., Schuster, W. J., \& Zhao, G. 2000, A\&A, 353, 722

Prochaska, J. X., \& McWilliam, A. 2000, ApJ, 537, L57

Reyniers, M. 2002, Ph.D. Thesis, K.U.Leuven

Reyniers, M., \& Van Winckel, H. 2001, A\&A, 365, 465

Reyniers, M., Van Winckel, H., Biémont, E., \& Quinet, P. 2002, A\&A, 395, L35 
Reyniers, M., Van Winckel, H., Gallino, R., \& Straniero, O. 2004, A\&A, 417, 269

Roberts, D. H., Lehar, J., \& Dreher, J. W. 1987, AJ, 93, 968

Rufener, F. 1981, A\&AS, 45, 207

Rufener, F., \& Nicolet, B. 1988, A\&A, 206, 357

Sackmann, I.-J., \& Boothroyd, A. I. 1999, ApJ, 510, 217

Sasselov, D. D. 1984, Ap\&SS, 102, 161

Sasselov, D. D. 1985, Inform. Bull. Var. Stars, 2776, 1

Schoenaers, C., \& Cuypers, J. 2004, in Variable Stars in the Local Group, ed. D. W. Kurtz, \& K. R. Pollard, ASP Conf. Proc., 310, 283

Schwarzenberg-Czerny, A. 1991, MNRAS, 253, 198

Stellingwerf, R. F. 1978, ApJ, 224, 953
Strohmeier, W., Knigge, R., \& Ott, H. 1965, Inform. Bull. Var Stars, 81,1

Travaglio, C., Galli, D., Gallino, R., et al. 1999, ApJ, 521, 691

Travaglio, C., Gallino, R., Arnone, E., et al. 2004, ApJ, 601, 864

Van Winckel, H. 1997, A\&A, 319, 561

Van Winckel, H. 2003, ARA\&A, 41, 391

Van Winckel, H., \& Reyniers, M. 2000, A\&A, 354, 135

Waelkens, C., \& Burnet, M. 1985, Inform. Bull. Var. Stars, 2808, 1

Wallerstein, G. 2002, PASP, 114, 689

Wasserburg, G. J., Boothroyd, A. I., \& Sackmann, I.-J. 1995, ApJ, 447, L37 